\title{
The Effects of Trajectory and Endpoint Errors in a Reaching Movement on the Sense of Agency
}

\author{
Takuro Zama1, Yoshiyuki Takahashi' ${ }^{1}$, Sotaro Shimada ${ }^{2 *}$ \\ ${ }^{1}$ Electrical Engineering Program, Graduate School of Science and Technology, Meiji University, Kawasaki-shi, Japan \\ ${ }^{2}$ Department of Electronics and Bioinformatics, School of Science and Technology, Meiji University, Kawasaki-shi, Japan \\ Email: *sshimada@meiji.ac.jp
}

\begin{abstract}
How to cite this paper: Zama, T., Takahashi, Y., \& Shimada, S. (2017). The Effects of Trajectory and Endpoint Errors in a Reaching Movement on the Sense of Agency. Psychology, 8, 2321-2332. https://doi.org/10.4236/psych.2017.814146
\end{abstract}

Received: November 2, 2017

Accepted: December 3, 2017

Published: December 6, 2017

Copyright ( 92017 by authors and Scientific Research Publishing Inc. This work is licensed under the Creative Commons Attribution International License (CC BY 4.0).

http://creativecommons.org/licenses/by/4.0/

\section{(c) (i) Open Access}

\begin{abstract}
The sense of agency (SoA) refers to the subjective feeling that an individual can control their own action with their own will. However, it is still unclear which aspects of the motor control process precisely affect the sense of agency. In this study, we investigated how the SoA is modulated by the online motor performance (trajectory error) and the outcome of the reaching movement (endpoint error). The results showed that the invalid priming and the visual feedback delay significantly increased both the trajectory and endpoint errors and that these errors significantly attenuated the SoA. The further correlation analyses showed that the decrease in SoA was significantly correlated with the trajectory error, but not with the endpoint error, when the error was explicitly noticed. We suggest that the deterioration in online motor performance, at least in a reaching movement, is the main cause of the attenuation in the SoA.
\end{abstract}

\section{Keywords}

Sense of Agency, Online Motor Control, Error Detection

\section{Introduction}

One of the fundamental aspects of self-body recognition is the feeling that a person's bodily movements are caused by themselves, which is called the sense of agency (SoA) (Gallagher, 2000). Previous studies have proposed the comparator model to explain the mechanism of the SoA (Blakemore et al., 2000; David et al., 2008). In this model, copies of motor commands (the efference copy) are sent to the "internal forward model" to predict the sensory feedback that will be acquired as the outcome of movement (Wolpert \& Ghahramani, 2000). The prediction is compared with the actual sensory feedback information, and the result 
of the comparison, known as the prediction error, is used to correct the movement error (i.e., feedback control). The SoA is said to occur if the prediction is consistent with the actual feedback. Any mismatch leads to a feeling "the movement is not me".

Previous studies have studied the extent to which spatial or temporal deviations in feedback attenuate the SoA (Blakemore et al., 1999; Farrer et al., 2003; Franck et al., 2001; Ismail \& Shimada, 2016; Sato \& Yasuda, 2005; Shimada et al., 2010; Van Den Bos \& Jeannerod, 2002; Wen et al., 2015a, 2015b, 2016, 2017). Shimada et al. (2010) inserted various time delays in the visual feedback to participants who were moving their hand. The authors instructed the participants to judge whether the observed hand movements were delayed with respect to their own movement. The results showed that participants began to notice the delay when it exceeded about 200 ms. Ismail and Shimada (2016) investigated the SoA against an observed hand that reflected participants' hand movements with various delays. The results indicated a decrease in the SoA if the visual feedback was delayed by $300 \mathrm{~ms}$ or more, suggesting that temporal consistency of less than about $200 \mathrm{~ms}$ is required for the occurrence of SoA. The temporal mismatch between actual movements and its online feedbacks would attenuate the SoA although the intended movement will be found to be achieved later.

The SoA occurs when the intended result is obtained by one's own action, but humans do not always feel in control of the outcome or their body, even if the intent is merely consistent with the outcome: one will not feel the SoA when he pitched away the garbage from the trash box if he had pushed by someone at the moment he threw it. The question we address here is to what extent the proximal action performance (e.g., trajectory of the throwing garbage) and the distal outcome (e.g., whether the garbage entered the trach box) affect the SoA, respectively. Although, recent studies have suggested that stronger SoA occurs by acquiring the intended outcome via the intended movement process (Caspar et al., $2015,2016)$, how the online movement process and the outcome affect SoA is largely uninvestigated.

In the present study, we investigate how the SoA is modulated by errors in movement control to achieve the intended outcome. In so doing, we employed a reaching task, which is performed by feed forward and feedback control. The feed forward control is driven mainly by using inverse model generating a motor command on the basis of the desired outcome; and the feedback control becomes active in the latter half of the movement to adjust and end the reaching motion (Desmurget \& Grafton, 2000; Osumi et al., 2017). We present spatial priming stimuli in either the identical (valid) or opposite (invalid) direction to the goal position before the movement. It is assumed that invalid priming biases the information of the goal position to the opposite direction at the stage of the initial trajectory determination. A motor command based on the incorrect trajectory determination should cause the feed forward control error (trajectory error). On the other hand, we introduced a delay in the visual feedback to induce a feedback control error in the reaching movement. When the trajectory error 
occurs, trajectory is recomputed and adjusted in real time (Todorov \& Jordan, 2002). However, if a delay is inserted to the visual feedback, this adjustment was generated based on the false feedback of the hand position and velocity, and the adjusted motor command will be wrong, which will cause the trajectory error, as well as the endpoint error, which represents the distance between the goal and the final hand position in the reaching movement. In summary, we introduced motor control errors in a reaching movement through the invalid priming and visual feedback delay, in order to investigate how online motor performance and outcome of the movement affect SoA.

\section{Material and Methods}

\subsection{Participants}

Seventeen healthy students with normal motor performance (two females, mean age \pm S.D. $=22.1 \pm 1.2$, range $20-25)$ participated in the experiment. According to Chapman's handedness inventory (Chapman \& Chapman, 1987), all subjects were right-handed (mean score 13.7, S.D. \pm 1.1 ). They had normal or corrected-to-normal visual acuity. Written informed consent was obtained from all participants. The experiments were approved by the ethics committee of the School of Science and Technology, Meiji University, and conducted according to the principles and guidelines of the Declaration of Helsinki.

\subsection{Apparatus}

The participants sat at a table with a 12.9 " touch panel display device $(2732 \times 2048$ resolution, $264 \mathrm{ppi}$, refresh rate $60 \mathrm{~Hz}$; iPad Pro, Apple, USA) and placed their right hand on the table (Figure 1). A double-sided mirror was installed above the table. The image of the touch panel and the participants' hands, which was reflected in the backside of the mirror, was filmed using a video camera (HDR-CX670, Sony, Japan). The filmed images were presented on a liquid-crystal monitor (LMD-232W, SONY, Japan) set above the mirror. Hence, the participants could see the reflected image of the touch panel display and their hands on the front side of the mirror. The angle of the mirror was finely adjusted before the experiment so that the touch panel display image appeared to the participants as if placed horizontally on the apparatus. Visual feedback delay was introduced using a hardware device (EDS3305, Eletex, Japan) connected between the video camera and the LCD monitor. The intrinsic delay of the visual feedback was approximately $88 \mathrm{~ms}$, as measured by a high-speed camera (EX-F1, CASIO, Japan).

\subsection{Procedure}

Participants performed a reaching task in which they were asked to swipe their right index finger from the home position to either the left or right side goal area displayed on the touch panel display device (Figure 2). As the priming stimulus of the goal direction, the edge of either the left or right goal area flashed red for a period of $33 \mathrm{~ms}$ some $2000 \mathrm{~ms}$ after the home position was tapped. Two kinds of priming condition were used: compatible direction (valid) and incompatible 


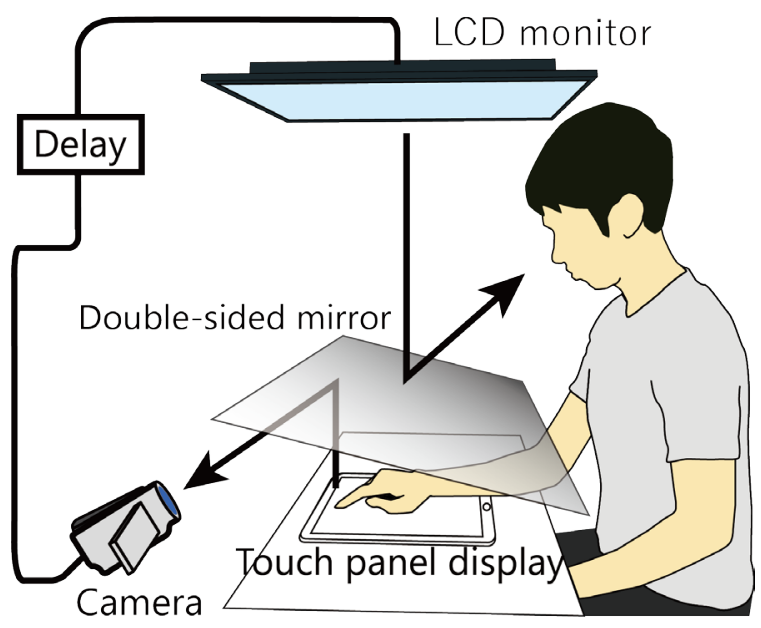

Figure 1. Experimental setup. Subjects conducted the reaching task on the touch panel display. Images of the hand and touch panel display were filmed by a camera via the back-side of the mirror. Visual feedback delay was inserted to the filmed image and participants observed the reflected image displayed on an LCD monitor.

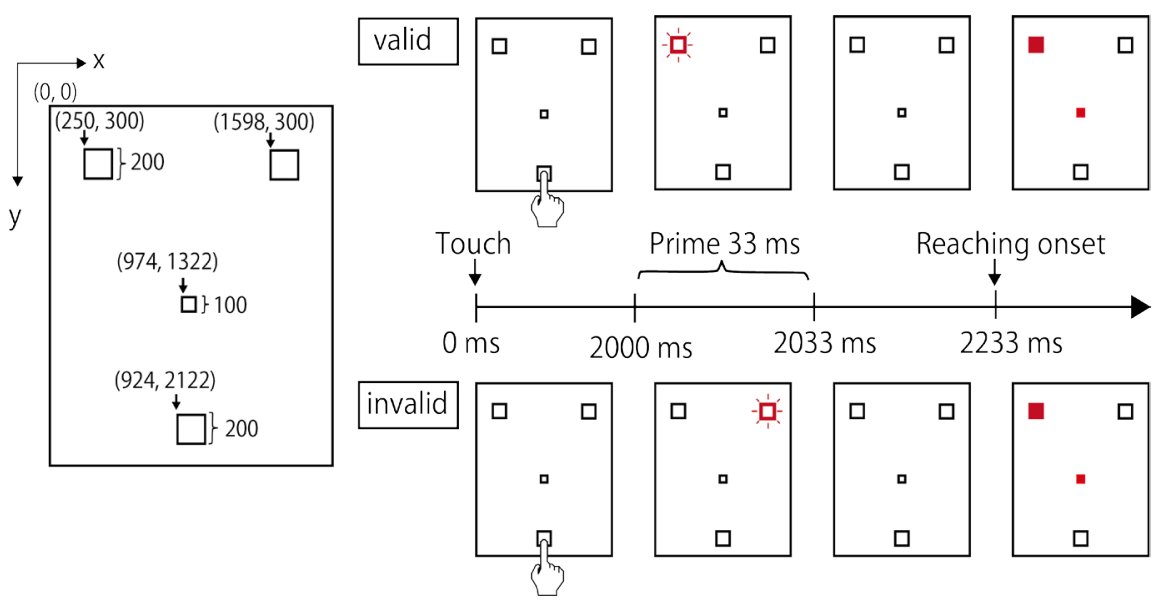

Figure 2. Schematic of trial procedure and stimuli. Participants were instructed to swipe their right index finger from the home position to either the left or right goal. Two seconds after touching the home position, a priming stimulus was displayed at identical (valid priming) or opposite (invalid priming) positions with respect to the goal. The goal area and the central square turned red $200 \mathrm{~ms}$ after the priming stimuli appeared, signifying the go cue. Participants were instructed to fixate on the central square and start movement as soon and as accurately as possible at the go cue.

direction (invalid) with respect to the goal direction. The central square and either the left/right goal position became red $200 \mathrm{~ms}$ after the priming stimulus, indicating the movement cue and goal position. Participants were instructed to fixate on the central square and start reaching toward the indicated goal position via the central square as soon and as accurately as possible after the go cue. The trajectory was not displayed on the touch panel display so that participants could not confirm the trajectory error after the task.

Each experiment consisted of four blocks of 56 trials, each with a 3-min inter-block interval. Seven visual feedback delay conditions were applied for each 
trial in random order: the delay ranged from 121 - $319 \mathrm{~ms}$ in 33-ms intervals. Valid- and invalid-priming conditions were randomly intermixed within each block at a 3:1 ratio. The number of trials was equal in the left- and right-reaching directions, and the order of the reaching direction was random. Therefore, each participant conducted a total of 224 reaching tasks.

Participants judged whether the observed hand image was exactly synchronized with their hand movement (i.e., whether the visual feedback delay was present or absent) and indicated the extent of their agreement or disagreement with an eight-statement questionnaire adopted from previous studies on SoA (Kalckert \& Ehrsson, 2012; Shimada et al., 2010) as following: 1) “The observed hand moved just like I wanted it to, as if it was obeying my will." 2) "I felt as if I was controlling the movements of the observed hand." 3) "I felt as if I was causing the movement I saw." 4) "Whenever I moved my hand, I expected the observed hand to move in the same way." Every question was rated on a 7-point Likert scale ranging from “ -3 ” (totally disagree) to “ +3 ” (totally agree), with " 0 ” indicating neither agreement nor disagreement.

\subsection{Data Analysis}

\subsubsection{Analysis of Delay-Detection Performance}

The detection probability of visual feedback delay for each delay condition and priming condition was calculated for each participant. To estimate each observer's delay detection threshold (DDT), representing the delay length at which the delay detection probability is $50 \%$, logistic curves were fitted to each participant's responses based on the following formula (Afraz et al., 2006; Shimada et al., 2010, 2014):

$$
P(t)=1 /\left(1+\exp \left\{-a\left(t-t_{D D T}\right)\right\}\right)
$$

where $t$ is the visual feedback delay length, $P(t)$ is the probability of making an asynchronous judgment, $a$ indicates the steepness of the fitted curve, and $t_{D D T}$ indicates the $D D T$. The fitting was performed using a nonlinear least-squares method with a trust-region algorithm provided by the Curve Fitting Toolbox in Matlab 9.1 (MathWorks, Natick, MA). In this study, $t$ served as an independent variable and $P(t)$ represented the observed data. Paired t-tests were conducted to the parameters $a$ and $t_{D D T}$ to investigate the influence of the priming stimuli on the delay-detection performance.

\subsubsection{Analysis of Reaching-Task Performance and SoA}

Movement onset and offset were determined as the times at which the hand velocities increased beyond and decreased below $10 \mathrm{~mm} / \mathrm{s}$, respectively (Messier \& Kalaska, 1999; Igarashi et al., 2011). The performance in the reaching task was evaluated by the endpoint error and the reaching trajectory error. The endpoint error was defined as the distance from the center of the goal area to the endpoint of each trial. The trajectory error was defined as the area enclosed by the trajec- 
tory of each trial and the mean trajectory in minimum-delay and valid- priming conditions, reflecting the error from the ideal trajectory traced under the minimum disturbance condition. We applied two-way (priming $\times$ delay) repeated-measure analyses of variance (ANOVA) separately to the endpoint error, trajectory error, and SoA as evaluated by the questionnaire.

\section{Result}

\subsection{Reaching Task Performance}

Some trajectories were attracted in the opposite direction to the goal in the invalid condition (Figure 3(a)). Figure $3(\mathrm{~b})$ shows the endpoint error averaged across all participants. Two-way ANOVA revealed significant main effects

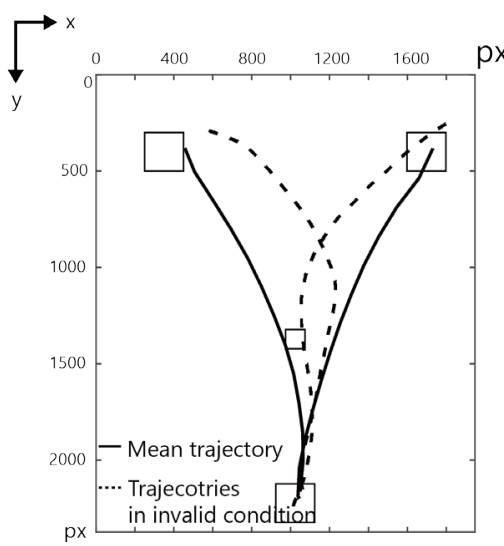

(a)

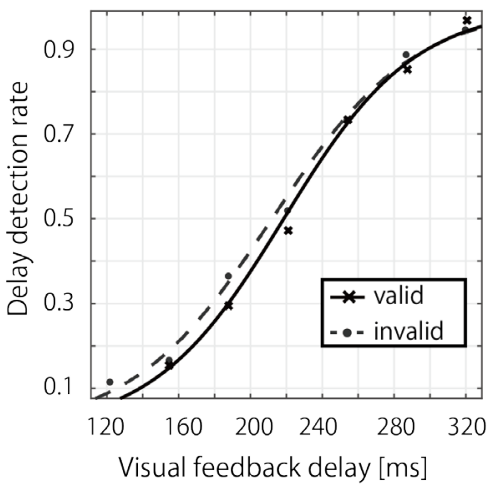

(d)

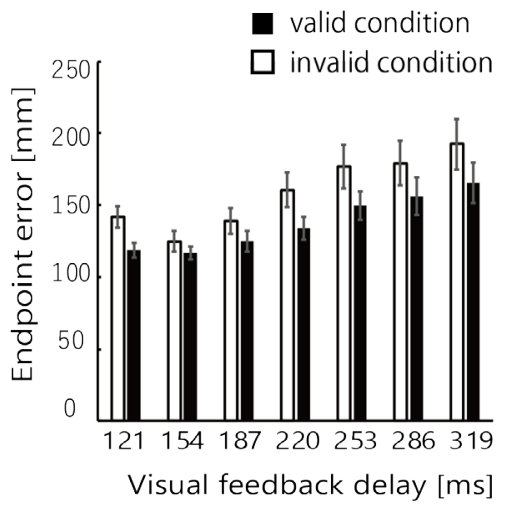

(b)

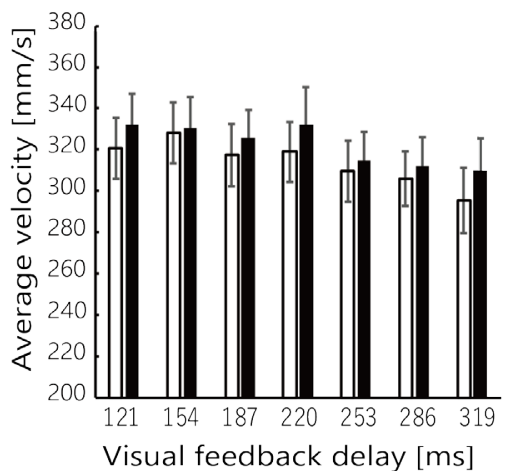

(e)

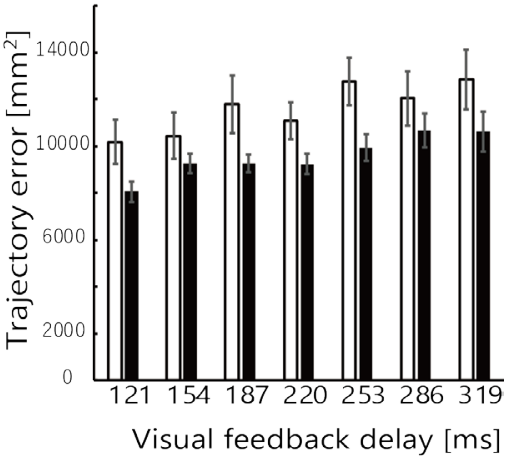

(c)

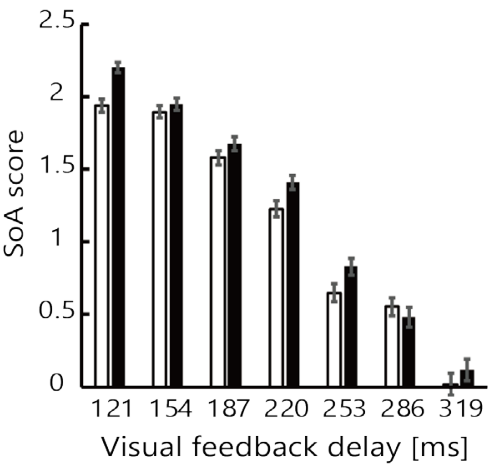

(f)

Figure 3. Motor performance, SoA score, and delay detection performance. (a) Typical trajectory of a participant. Black lines denote trajectories averaged across those under the minimum delay and valid condition. The broken lines denote trajectories in the invalid priming condition. Trajectories in the invalid priming condition are biased in the opposite direction with respect to the goal; (b) Endpoint error, defined as the distance from the center of the goal to an endpoint; (c) Trajectory error, defined as the area enclosed by a trajectory of each trial and the mean trajectory; (d) Delay detection curves for valid and invalid priming conditions averaged across all participants. The delay detection threshold, representing the delay length at which the delay detection probability is $50 \%$, was decreased by the invalid priming stimulus; (e) Average velocity, calculated by averaging the velocity from movement onset to end of the movement; (f) SoA score, calculated by averaging the questionnaire ratings across items 1 - 4 . (b) (c) (e) (f) Two-way (priming $\times$ delay) repeated-measure ANOVA showed the main effects of priming and delay in the movement error and SoA score. In all indices, the black bar denotes data from the valid condition, whereas the white bar indicates data from the invalid condition. All error bars denote standard error. 
caused by priming $\left(F(1,16)=24.31, p<0.001, \eta_{p}^{2}=0.60\right)$ and delay $(F(6,96)$ $\left.=12.00, p<0.001, \eta_{p}^{2}=0.43\right)$. There was no significant interaction between these factors $\left(F(6,96)=0.60, p=0.73, \eta_{p}^{2}=0.04\right)$. Subsequent analysis showed that the arrival-point errors for delays of 121,154 , and 187 ms were significantly smaller than those for delays of 253,286, and $319 \mathrm{~ms}$, and the endpoint error for delays of $220 \mathrm{~ms}$ was smaller than that of $319 \mathrm{~ms}$ (Tukey's honestly significant difference $(H S D)$ test, $p<0.05)$. Figure $3(c)$ depicts the grand-averaged trajectory error for the valid and invalid conditions. Two-way ANOVA revealed significant main effects caused by priming $\left(F(1,16)=11.35, p<0.01, \eta_{p}^{2}=0.42\right)$ and delay $\left(F(6,96)=3.34, p<0.01, \eta_{p}^{2}=0.17\right)$. There was no significant interaction between these factors $\left(F(6,96)=0.67, p=0.67, \eta_{p}^{2}=0.04\right)$. Subsequent analysis showed that the trajectory error for delays of $121 \mathrm{~ms}$ was significantly smaller than those for delays of 253, 286, and 319 ms (Tukey's HSD test, $p<$ 0.05). In consideration of the effect of speed accuracy tradeoff, we calculated the reaction time (RT), and applied ANOVA to the average velocity like other behavior date. The averaged RT for valid condition was $555 \mathrm{~ms}$, while the averaged RT for invalid condition was $565 \mathrm{~ms}$. There was no significant difference between the RTs $(t(16)=-1.80, p=0.09)$. The two-way ANOVA for average velocity showed the significant main effect on delay $\left(F(6,96)=8.10, p<0.001, \eta_{p}^{2}\right.$ $=0.34$ ), indicating that the average velocity became slowly as increasing visual feedback delay. We did not find any other significant main effect or interaction $(p>0.05)$.

\subsection{Discrimination Threshold of Visual Feedback Delay}

For all participants, the probability of delay detection became larger as the visual feedback delay increased in both the valid and invalid conditions (Figure 3(d)). The fitted curves for delay-detection probabilities show that the DDT of invalid conditions was significantly shorter than that of valid conditions $(t(16)=-2.54$, $p<0.05)$ : the DDTs of invalid/valid conditions were 209.9 and $216.7 \mathrm{~ms}$, respectively. There was no significant difference between the slopes of the curves of valid and invalid conditions $(t(16)=1.25, p=0.23)$.

\subsection{SoA}

For the averaged ratings of the questionnaire items, there were significant main effects caused by priming $\left(F(1,16)=7.64, p<0.05, \eta_{p}^{2}=0.32\right)$ and delay $(F(6$, $\left.96)=45.30, p<0.001, \eta_{p}^{2}=0.74\right)$, and no significant interaction between these factors $\left(F(6,96)=1.90, p=0.089, \eta_{p}^{2}=0.11\right.$ ) (Figure $3(\mathrm{f})$ ). Subsequent analysis (Tukey's HSD test) showed that there were significant differences within all pairs of conditions, except between the pairs of 121 and $154 \mathrm{~ms}, 154$ and $187 \mathrm{~ms}$, 187 and $220 \mathrm{~ms}, 253$ and 286 , and 286 and $319 \mathrm{~ms}(p<0.05)$.

\subsection{Correlation between SoA and Detection of Motor Control Error}

The SoA significantly decreased when the delay length exceeded 220 ms which 
was near the DDT. To examine the factors that impacted SoA, we carried out Pearson's correlation analysis between the delay detection rate and the SoA. The result showed s significant correlation $\left(r^{2}=0.36, p<0.01\right)$, indicated that the SoA decreased when participants explicitly noticed the visual feedback delay (Figure 4).

Further, we applied correlation analyses using only the trials in which participants explicitly noticed the delay. We found a significant correlation of the SoA with the trajectory error $\left(r^{2}=0.18, p<0.01\right.$, Bonferroni-corrected) (Figure $5(\mathrm{a}))$, but not with the endpoint error $\left(r^{2}=0.06, p=0.21\right.$, Bonferroni-corrected $)$ (Figure 5(b)).

\section{Discussion}

In this study, we investigated how the SoA is modulated by the online motor

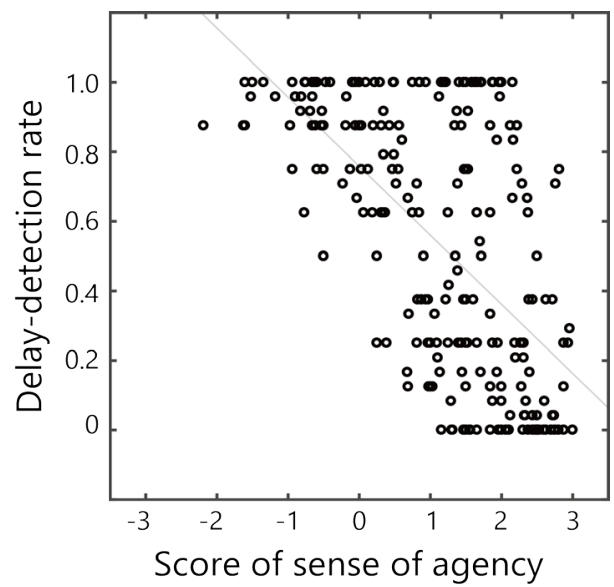

Figure 4. Correlation analysis between the SoA and the delay-detection rate. The delaydetection rate significantly correlated with the sense of agency $\left(r^{2}=0.36, p<0.01\right)$.

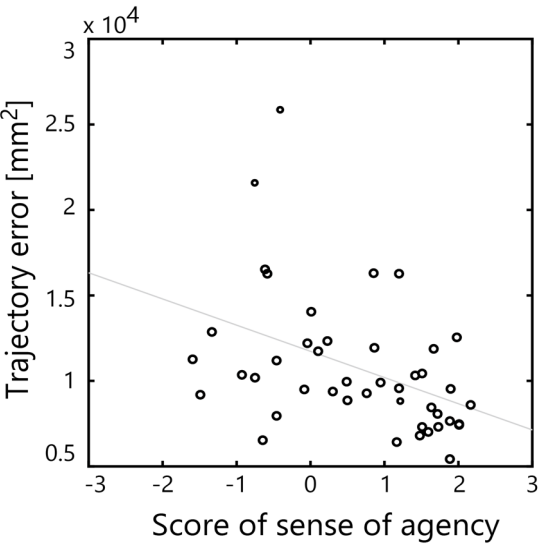

(a)

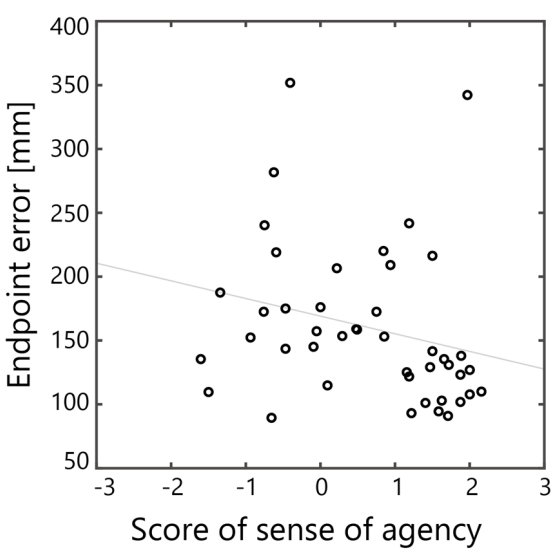

(b)

Figure 5. Correlation analyses between the SoA and motor performance. (a) The significant correlation between the sense of agency and the trajectory errors $\left(r^{2}=0.18, p<0.01\right)$; (b) The non-significant correlation between the sense of agency and the endpoint errors $\left(r^{2}=0.06, p=0.21\right)$. 
performance (trajectory error) and the outcome of the reaching movement (endpoint error). The results showed that the invalid priming and the visual feedback delay significantly increased both the trajectory and endpoint errors and that these errors significantly attenuated the SoA. The further correlation analyses showed that the decrease in SoA was significantly correlated with the trajectory error, but not with the endpoint error, when the error was explicitly noticed. We suggest that the deterioration in online motor performance, at least in a reaching movement, is the main cause of the attenuation in the SoA.

Our results that the SoA decreased when the visual feedback delay exceeded about $200 \mathrm{~ms}$ is consistent with various previous studies (Franck et al., 2001; Ismail \& Shimada, 2016; Sato \& Yasuda, 2005; Shimada et al., 2010; Wen et al., 2015a, 2015b, 2016, 2017). Wen et al. (2017) instructed participants to move a cursor to a goal by pushing control buttons. The cursor responded with various delay. The structural equation modeling showed the causal relation that the SoA decreased by detecting the delay. Shimada et al. (2010) inserted various time delays in the visual feedback to participants who were moving their hand. The authors instructed the participants to judge whether the observed hand movements were delayed with respect to their own movement. The results showed that participants began to notice the delay when it exceeded about $230 \mathrm{~ms}$. Ismail and Shimada (2016) investigated the SoA during observations of a virtual hand movement that reflected participants' own hand. They found that the SoA decreased when the visual feedback delay of the hand movement exceeded $300 \mathrm{~ms}$. These findings suggest that the SoA would be maintained when the temporal mismatch between the actual movement and its sensory feedback is less than 200 ms.

Previous studies have demonstrated that conceptual priming affects the SoA (Aarts et al., 2005; Chambon \& Haggard, 2012; Damen et al., 2014; Moore et al., 2009; Sato, 2009; Sidarus et al., 2013; Wenke et al., 2010). Sato (2009) presented the word "blue" or "red" as a prime stimulus just before the participant made a button-press to cause a blue or red circle to be appeared on the monitor, and found that the SoA was enhanced by the conceptual congruency between the prime word and the action effect. Other studies showed that the fluent action selection by priming enhanced the feeling of control over the action outcome, even when the prime did not improve the predictability of the outcome (Chambon \& Haggard, 2012; Wenke et al., 2010). Our study expands the previous findings in that the SoA is also affected by sensorimotor priming, rather than conceptual priming. It should be noticed that there was no significant difference in RTs between the valid and invalid conditions, indicating that the SoA was not modulated by influent action selection.

Synofzik et al. (2008) proposed a two-step account of agency consisting of the "feeling of agency (FoA)" and "judgement of agency (JoA)": the FoA is the nonconceptual feeling of being the agent at the sensorimotor level, which merely reflects whether the action was self-caused or not. In case there exists a mismatch 
between an action and its sensory feedback, we experience the action as strange, peculiar and not fully done by me, and then the sense of agency is further investigated by with conceptual factors like intentions, contextual cues, and social cues, which resulted in JoA. Several previous studies have demonstrated that the JoA occurs when it is retrospectively confirmed that the outcome of action was consistent with the desired or expected outcome (Farrer et al., 2013; Sato \& Yasuda, 2005; Wegner et al., 2004; Wen et al., 2015a). In this study, however, the endpoint errors, which reflect the final outcome of a reaching movement and hence relevant to JoA, did not affect SoA. This indicates that JoA is not a critical factor for SoA in our experiment. Rather, attenuation of SoA was significantly correlated with the amount of the trajectory error, which reflects errors in online motor control process, and hence presumably relevant with FoA. Thus, we consider that SoA in a reaching movement arises mainly based on FoA, not on JoA, during online motor processing.

\section{Conclusion}

This study aimed to examine which aspects of the motor control process precisely affect the sense of agency (SoA). Our results showed that the invalid priming of goal position and visual feedback delay increased both trajectory and endpoint errors in a reaching movement, which eventually decreased the SoA. We suggest that the SoA, at least in a reaching movement, is largely affected by explicit awareness of sensory prediction errors used in the online motor control process, rather than inconsistency between the desired goal and the final outcome. Our novel technique can contribute to elucidating of the mechanism of "feeling of agency" disability on self-body.

\section{Acknowledgements}

This work was partially supported by Grants-in-Aid for Scientific Research (16H02839 and 17H05915) from the Japan Society for the Promotion of Science (JSPS).

\section{References}

Aarts, H., Custers, R., \& Wegner, D. M. (2005). On the Inference of Personal Authorship: Enhancing Experienced Agency by Priming Effect Information. Consciousness and Cognition, 14, 439-458. https://doi.org/10.1016/j.concog.2004.11.001

Afraz, S.-R., Kiani, R., \& Esteky, H. (2006). Microstimulation of Inferotemporal Cortex Influences Face Categorization. Nature, 442, 692-695. https://doi.org/10.1038/nature04982

Blakemore, S. J., Frith, C. D., \& Wolpert, D. M. (1999). Spatio-Temporal Prediction Modulates the Perception of Self-Produced Stimuli. Journal of Cognitive Neuroscience, 11, 551-559. https://doi.org/10.1162/089892999563607

Blakemore, S. J., Wolpert, D., \& Frith, C. (2000). Why Can't You Tickle Yourself? Neuroreport, 11, R11-R16. https://doi.org/10.1097/00001756-200008030-00002

Caspar, E. A., Cleeremans, A., \& Haggard, P. (2015). The Relationship between Human 
Agency and Embodiment. Consciousness and Cognition, 33, 226-236. https://doi.org/10.1016/j.concog.2015.01.007

Caspar, E. A., Desantis, A., Dienes, Z., Cleeremans, A., \& Haggard, P. (2016). The Sense of Agency as Tracking Control. PLoS ONE, 11, 1-16.

https://doi.org/10.1371/journal.pone.0163892

Chambon, V., \& Haggard, P. (2012). Sense of Control Depends on Fluency of Action Selection, Not Motor Performance. Cognition, 125, 441-451. https://doi.org/10.1016/j.cognition.2012.07.011

Chapman, L. J., \& Chapman, J. P. (1987). The Measurement of Handedness. Brain and Cognition, 6, 175-183. https://doi.org/10.1016/0278-2626(87)90118-7

Damen, T. G. E., van Baaren, R. B., \& Dijksterhuis, A. (2014). You Should Read This! Perceiving and Acting upon Action Primes Influences One's Sense of Agency. Journal of Experimental Social Psychology, 50, 21-26. https://doi.org/10.1016/j.jesp.2013.09.003

David, N., Newen, A., \& Vogeley, K. (2008). The "Sense of Agency" and Its Underlying Cognitive and Neural Mechanisms. Consciousness and Cognition, 17, 523-534. https://doi.org/10.1016/j.concog.2008.03.004

Desmurget, M., \& Grafton, S. (2000). Forward Modeling Allows Feedback Control for Fast Reaching Movements. Trends in Cognitive Sciences, 4, 423-431. https://doi.org/10.1016/S1364-6613(00)01537-0

Farrer, C., Franck, N., Georgieff, N., Frith, C. D., Decety, J., \& Jeannerod, M. (2003). Modulating the Experience of Agency: A Positron Emission Tomography Study. NeuroImage, 18, 324-333. https://doi.org/10.1016/S1053-8119(02)00041-1

Farrer, C., Valentin, G., \& Hupé, J. M. (2013). The Time Windows of the Sense of Agency. Consciousness and Cognition, 22, 1431-1441.

https://doi.org/10.1016/j.concog.2013.09.010

Franck, N., Farrer, C., Georgieff, N., Marie-Cardine, M., Dalery, J., D’Amato, T., \& Jeannerod, M. (2001). Defective Recognition of One's Own Actions in Patients with Schizophrenia. American Journal of Psychiatry, 158, 454-459.

https://doi.org/10.1176/appi.ajp.158.3.454

Gallagher, S. (2000). Philosophical Conceptions of the Self: Implications for Cognitive Science. Trends in Cognitive Sciences, 4, 14-21.

https://doi.org/10.1176/appi.ajp.158.3.454

Igarashi, K., Shibuya, S., Sano, H., Takahashi, M., Satomi, K., \& Ohki, Y. (2011). Functional Assessment of Proximal Arm Muscles by target-Reaching Movements in Patients with Cervical Myelopathy. Spine Journal, 11, 270-280.

https://doi.org/10.1016/j.spinee.2011.02.003

Ismail, M. A. F., \& Shimada, S. (2016). "Robot" Hand Illusion under Delayed Visual Feedback: Relationship between the Senses of Ownership and Agency. PLoS ONE, 11, 1-9. https://doi.org/10.1371/journal.pone.0159619

Kalckert, A., \& Ehrsson, H. H. (2012). Moving a Rubber Hand That Feels Like Your Own: A Dissociation of Ownership and Agency. Frontiers in Human Neuroscience, 6, 1-14. https://doi.org/10.3389/fnhum.2012.00040

Messier, J., \& Kalaska, J. F. (1999). Comparison of Variability of Initial Kinematics and Endpoints of Reaching Movements. Experimental Brain Research, 125, 139-152. https://doi.org/10.1007/s002210050669

Moore, J. W., Wegner, D. M., \& Haggard, P. (2009). Modulating the Sense of Agency with External Cues. Consciousness and Cognition, 18, 1056-1064.

https://doi.org/10.1016/j.concog.2009.05.004 
Osumi, M., Sumitani, M., Kumagaya, S., \& Morioka, S. (2017). Optimal Control of Reaching Is Disturbed in Complex Regional Pain Syndrome: A Single-Case Study. Journal of Pain Research, 10, 167-173. https://doi.org/10.2147/JPR.S118846

Sato, A., \& Yasuda, A. (2005). Illusion of Sense of Self-Agency: Discrepancy between the Predicted and Actual Sensory Consequences of Actions Modulates the Sense of Self-Agency, But Not the Sense of Self-Ownership. Cognition, 94, 241-255. https://doi.org/10.1016/j.cognition.2004.04.003

Sato, A. (2009). Both Motor Prediction and Conceptual Congruency between Preview and Action-Effect Contribute to Explicit Judgment of Agency. Cognition, 110, 74-83. https://doi.org/10.1016/j.cognition.2008.10.011

Shimada, S., Qi, Y., \& Hiraki, K. (2010). Detection of Visual Feedback Delay in Active and Passive Self-Body Movements. Experimental Brain Research, 201, 359-364. https://doi.org/10.1007/s00221-009-2028-6

Shimada, S., Suzuki, T., Yoda, N., \& Hayashi, T. (2014). Relationship between Sensitivity to Visuotactile Temporal Discrepancy and the Rubber Hand Illusion. Neuroscience Research, 85, 33-38. https://doi.org/10.1016/j.neures.2014.04.009

Sidarus, N., Chambon, V., \& Haggard, P. (2013). Priming of Actions Increases Sense of Control over Unexpected Outcomes. Consciousness and Cognition, 22, 1403-1411. https://doi.org/10.1016/j.concog.2013.09.008

Synofzik, M., Vosgerau, G., \& Newen, A. (2008). Beyond the Comparator Model: A Multifactorial Two-Step Account of Agency. Consciousness and Cognition, 17, 219-239. https://doi.org/10.1016/j.concog.2007.03.010

Todorov, E., \& Jordan, M. I. (2002). Optimal Feedback Control as a Theory of Motor Coordination. Nature Neuroscience, 5, 1226-1235. https://doi.org/10.1038/nn963

Van Den Bos, E., \& Jeannerod, M. (2002). Sense of Body and Sense of Action Both Contribute to Self-Recognition. Cognition, 85, 177-187. https://doi.org/10.1016/S0010-0277(02)00100-2

Wegner, D. M., Sparrow, B., \& Winerman, L. (2004). Vicarious Agency: Experiencing Control over the Movements of Others. Journal of Personality and Social Psychology, 86, 838-848. https://doi.org/10.1037/0022-3514.86.6.838

Wen, W., Yamashita, A., \& Asama, H. (2015a). The Influence of Action-Outcome Delay and Arousal on Sense of Agency and the Intentional Binding Effect. Consciousness and Cognition, 36, 87-95. https://doi.org/10.1016/j.concog.2015.06.004

Wen, W., Yamashita, A., \& Asama, H. (2015b). The Sense of Agency during Continuous Action: Performance Is More Important than Action-Feedback Association. PLoS ONE, 10, 1-16. https://doi.org/10.1371/journal.pone.0125226

Wen, W., Yamashita, A., \& Asama, H. (2016). Divided Attention and Processes Underlying Sense of Agency. Frontiers in Psychology, 7, 1-8.

Wen, W., Yamashita, A., \& Asama, H. (2017). The Influence of Performance on Action-Effect Integration in Sense of Agency. Consciousness and Cognition, 53, 89-98. https://doi.org/10.1016/j.concog.2017.06.008

Wenke, D., Fleming, S. M., \& Haggard, P. (2010). Subliminal Priming of Actions Influences Sense of Control over Effects of Action. Cognition, 115, 26-38. https://doi.org/10.1016/j.cognition.2009.10.016

Wolpert, D. M., \& Ghahramani, Z. (2000). Computational Principles of Movement Neuroscience. Nature Neuroscience, 3, 1212-1217. https://doi.org/10.1038/81497 